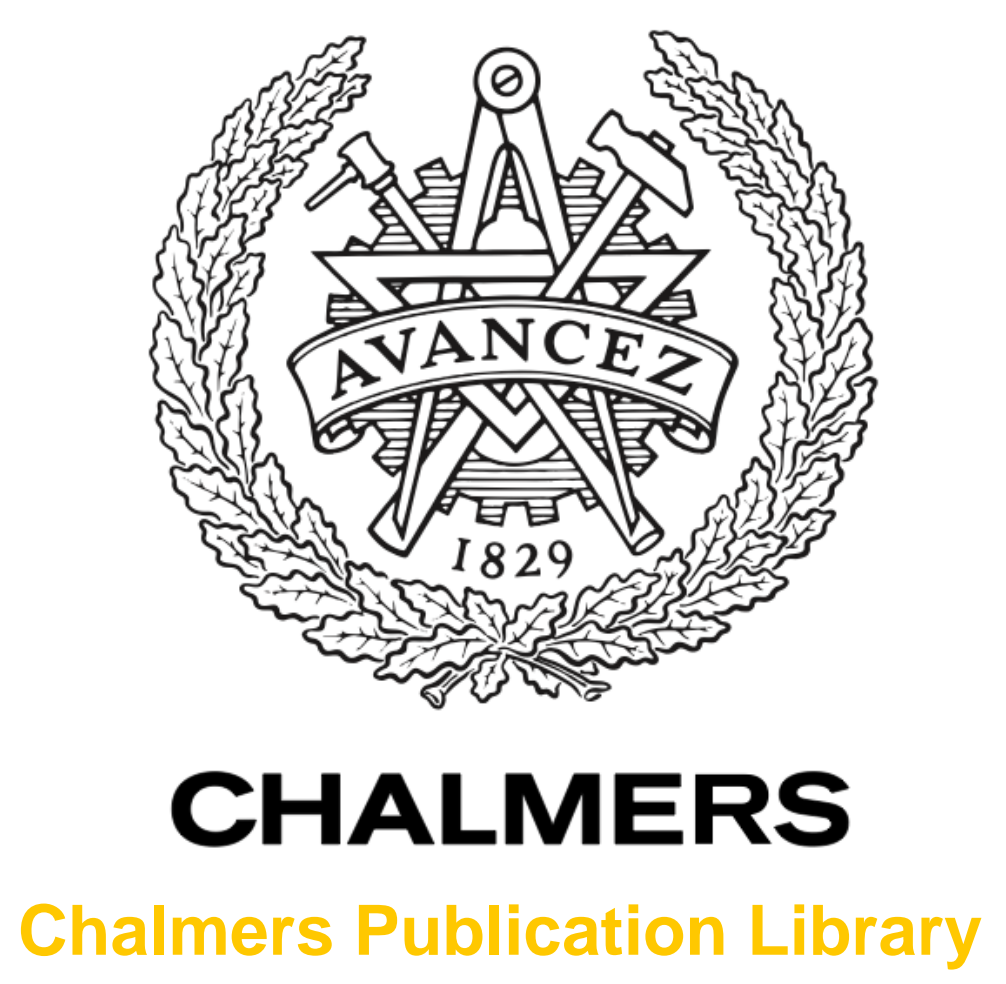

\title{
Suppression of Parallel Plate Modes in Low Frequency Microstrip Circuit Packages Using Lid of Printed Zigzag Wires
}

This document has been downloaded from Chalmers Publication Library (CPL). It is the author's version of a work that was accepted for publication in:

IEEE Microwave and Wireless Components Letters (ISSN: 1531-1309)

Citation for the published paper:

Rajo-Iglesias, E. ; Pucci, E. ; Kishk, A. (2013) "Suppression of Parallel Plate Modes in Low Frequency Microstrip Circuit Packages Using Lid of Printed Zigzag Wires". IEEE

Microwave and Wireless Components Letters, vol. 23(7), pp. 359-361.

http://dx.doi.org/10.1109/lmwc.2013.2265257

Downloaded from: http://publications.lib.chalmers.se/publication/180969

Notice: Changes introduced as a result of publishing processes such as copy-editing and formatting may not be reflected in this document. For a definitive version of this work, please refer to the published source. Please note that access to the published version might require a subscription.

Chalmers Publication Library (CPL) offers the possibility of retrieving research publications produced at Chalmers University of Technology. It covers all types of publications: articles, dissertations, licentiate theses, masters theses, conference papers, reports etc. Since 2006 it is the official tool for Chalmers official publication statistics. To ensure that Chalmers research results are disseminated as widely as possible, an Open Access Policy has been adopted.

The CPL service is administrated and maintained by Chalmers Library. 


\title{
Suppression of parallel plate modes in low frequency microstrip circuit packages using lid of printed zigzag wires
}

\author{
Eva Rajo-Iglesias, Senior Member, IEEE, Elena Pucci, Ahmed Kishk, Fellow Member, IEEE, Per-Simon \\ Kildal Fellow Member, IEEE,
}

\begin{abstract}
This work deals with the suppression of parallel plate and cavity modes in shielded microstrip circuits operating at the lower microwave frequency range. The suppression is achieved by using a lid made of zigzag wires printed periodically on narrow slices of ungrounded circuit boards, located vertically side by side. This structure is very compact both in periodicity and height, it suppresses cavity modes over about an octave 2:1 bandwidth, and it does not interfere with the packaged microstrip circuit.
\end{abstract}

Index Terms-AMC, parallel plate mode, gap waveguide

\section{INTRODUCTION}

The recently introduced gap waveguide technology [1] has opened for new applications of Artificial Magnetic Conductors (AMC) and other types of periodic surfaces in the area of packaging of microwave circuits. Previously, the authors have demonstrated how to use the bed of nails [2] for this purpose, suppressing cavity modes over a wide bandwidth [3]. There have also been presented other AMC-type structures that can be used for creating the stop band needed in gap waveguide applications [4], such as mushroom surfaces [5].

The bed of nails [2] has a thickness between 0.15 and $0.5 \lambda$ and is therefore useful at high frequencies where the pin lengths are physically short. However, when scaling them to frequencies below $10 \mathrm{GHz}$, the structure becomes too bulky even for tilted versions of it [6]. Previously, we have proposed to transform the bed of nails to a bed of springs [7] to realize the stop band with a more compact periodic surface. However, the fabrication of springs and mounting of them side by side is not easy and can be expensive. The length of the wire used in each spring should be approximately $0.25 \lambda$ in order to achieve the stopband function when many of them are located side by side.

In the present work we propose a printed zigzag version of a spring, and we test out how well it works for suppressing cavity modes when packaging a microstrip circuit example around $5 \mathrm{GHz}$. The structure is shown in Fig. 1 where all the design parameters are described. The periodic zigzag structure

Manuscript received January 1, 2013; revised March 3, 2013. This work has been supported in part by the Swedish research council VR and also by the Spanish government under projects TEC2010-20841-C04-01 and CONSOLIDER-INGENIO CSD2008-00068 (TERASENSE).

E. Rajo-Iglesias is with the Signal Theory and Communication Dpt., University Carlos III of Madrid, Spain (e-mail:eva@tsc.uc3m.es).

E. Pucci and P-.S. Kildal are with Signals and Systems Dpt., Chalmers University of Technology, Sweden.

A. Kishk is now with Concordia University, Canada is printed on several slices of thin dielectric boards, and these boards are located vertically side by side to form the periodic surface as shown in Fig. 1.a. The zigzags have electrical contacts with the bottom plate. We should also point out that the structure could be made of solid metal instead of printing it on a dielectric slab. However, for a moderate to low frequency range, the option of printing is more convenient from manufacturing point of view and does not add important losses. We should also mention that the literature even describes other wideband solutions for packaging, but they are mainly based on multilayer structures [8],[9] that are parallel with the microstrip circuit board or they are narrower band solutions as [10], [11].

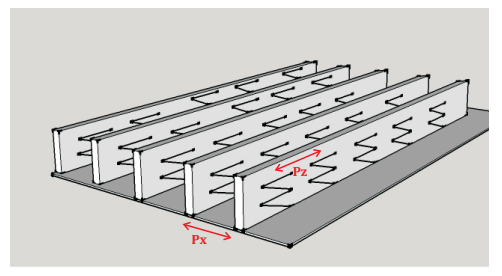

(a) Perspective

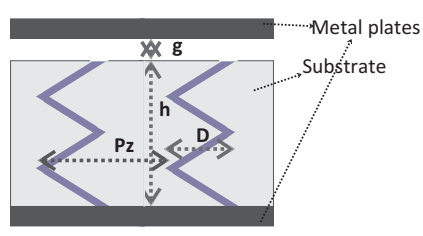

(b) Front view
Fig. 1. The zigzag structure with all the main geometrical parameters. The upper metal plate is actually the ground plane of the microstrip circuit board which we shall package. The substrate of the microstrip circuit board is not shown, but will in practice be located within the gap of height $\mathrm{g}$.

\section{PARAMETRIC STUdY}

First we determine the stopband of the parallel-plate modes by computing the dispersion diagram using a unit cell of the periodic grounded zigzag structure between parallel metal plates, as shown in Fig. 1.b, by using the eigenmode solver of CST Microwave Studio. The zigzag wires are grounded to the lower metal plate, and there is a gap between the zigzag structure and the upper metal surface inside which the microstrip circuit to be packed shall be located. The upper plate is actually the ground plane of the microstrip circuit. From the computations, we can conclude that the proposed structure has the folowing intrinsic limits for the stop band: the lower cutoff frequency appears when the electrical length of the wires is approximately $0.25 \lambda$ in free space, and the upper one appears when the spacing $h+g$ between the two metal plates is approximately $0.5 \lambda$ effectively. However, we must in addition require that the total length of the wire is smaller than $0.5 \lambda$ to achieve a stopband. This limit normally appears before the limit defined by $h+g$ smaller than $0.5 \lambda$. 
There is an important difference when comparing the zigzag structure to the bed of nails: the size of the gap $g$ is hardly affecting the lower cutoff frequency of the stopband. The reason comes from the fact that there is almost no capacitive effect between the top of the zigzag wires and the upper plate, in contrast to the capacitive effect created by the larger flat top of the pins, which is critical and strongly contributes to decrease the lower limit of the stop band [4]. This is an advantage because we can increase the space between the top of the zigzag and the circuit board to be packaged without affecting too much the stop band. On the other hand, bandwidth cannot be increased by using these capactive effects. educing the total length of the "wire" (i.e., reducing the diameter $D$, reducing the number of arms $N$ or reducing the height $h$ ) moves the complete stop band up in frequency.

There are two periods in the structure: one between the neighboring zigzags printed on the same layer $\left(P_{z}\right)$, and the other between neighboring slabs $\left(P_{x}\right)$. These periods do not need to be identical. We have used sub-wavelengths periods in all the examples considered, and we could not observe any difference in the stopband. The main requirement is that the number of zigzags or their size must create a dense enough lattice with a period smaller than $0.5 \lambda$ at all frequencies within the stop band.

\section{PROTOTYPE DESIGN}

We use the same simple microstrip circuit demonstrator as in [3], i.e. microstrip line with two $90^{\circ}$ bends. The targeted frequency range is from 3 to $6 \mathrm{GHz}$, and the microstrip circuit board has dimensions $55 \mathrm{~mm} \times 100 \mathrm{~mm}$. The line width is $1 \mathrm{~mm}$ and it is printed on a dielectric material with relative permittivity $\epsilon_{r}=4.5$ and $t 0.5 \mathrm{~mm}$ thickness (providing a 50 $\Omega$ line impedance). The line is not affected by the packaging as the cavity modes as discussed in [12]. The bends are known to cause radiation and excite cavity modes. However, at the frequencies we have selected and with the substrate thickness we have considered, the radiation will be almost negligible, and thus we expect to observe only weak excitation of cavity modes. In real circuits there may be many other causes for cavity mode excitations, such as non-linear amplifiers.

Considering this, we have chosen the following parameters for the unit cell, a zigzag unit cell with a number of sections of $N=3$ and $p_{x}=p_{z}=10 \mathrm{~mm}, D=7 \mathrm{~mm}, h=10 \mathrm{~mm}$ and $g$ $=2 \mathrm{~mm}$. The dispersion diagrams in the two main directions, i.e., the one parallel to the slabs and the one perpendicular to it are shown in Fig. 2. They have been computed including the substrate layer for the circuit located in between the gap and the upper plate according to Fig. 1.b.

We have numerically computed the frequencies of the cavity modes by using the eigenmode solver of CST Microwave Studio for two cases; i) empty cavity with smooth metal lid and depth equal to $h+g$ and, ii) the same cavity with the dielectric slabs without the printed zigzag shaped wires. The results for the first 8 modes are presented in Table I. Some of these modes have resonated within the stop band of the structure and consequently leads to its suppression.

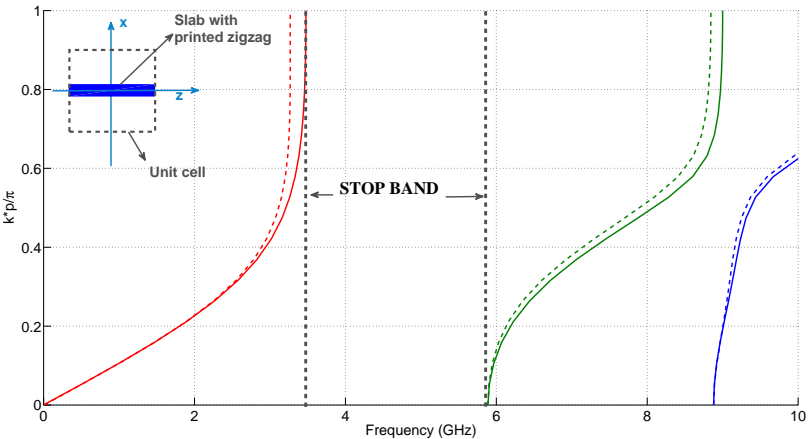

Fig. 2. Dispersion diagram of the designed zigzag structure in Fig. 1.b in the two main directions: perpendicular and parallel to the slabs. The inset represents the top view of the unit cell. Solid lines represent modes for $\hat{z}$ direction and dashed lines represent modes for $\hat{x}$ direction. In both cases: $N$ $=3, p_{x}=p_{z}=10 \mathrm{~mm}, D=7 \mathrm{~mm}, h=10 \mathrm{~mm}$ and $g=2 \mathrm{~mm}$.

TABLE I

FREQUENCIES OF THE FIRST 8 CAVITY MODES (IN GHZ) IN TWO PARTIALLY FILLED CAVITIES WITH SIZE $100 \times 55 \times 12 \mathrm{MM}$

\begin{tabular}{|c|c|c|c|c|c|c|c|c|}
\hline $\begin{array}{c}\text { empty } \\
\text { cavity }\end{array}$ & 3.06 & 3.98 & 5.17 & 5.56 & 6.11 & 6.47 & 6.94 & 7.83 \\
\hline $\begin{array}{c}\text { cavity } \\
\text { with slabs }\end{array}$ & 2.87 & 3.74 & 4.85 & 5.21 & 5.73 & 6.07 & 6.51 & 7.35 \\
\hline
\end{tabular}

\section{EXPERIMENTAL RESULTS}

The manufactured zigzag lid is shown in Fig. 3.b including the double-bent microstrip line to be packaged (fed by coaxial SMA connectors). The comparison is made with a smooth metal lid located at the same distance to the circuit as the plate to which the zigzag wires are soldered, i.e. $(h+g)$. The $S$-parameters are referred to the coaxial ports, so the imperfections of the coaxial to microstrip transitions are included in the presented results.

The experimental results are shown in Fig. 3.a. As expected the cavity modes are not excited very strongly for this transmission line at this frequency, but nevertheless we can observe cavity mode resonances when carefully studying the results and some of these resonances coincide with the ones in Table I. We show a detailed comparison of the $\left|S_{21}\right|$ parameters for two different zigzag lids, with the only difference being the material used to print the zigzag: one is printed on lossy FR4 (Fiber glass with $t g \delta=0.015$ ), and the other on lowloss Duroid $(\operatorname{tg} \delta=0.002)$. For both cases the cavity modes are clearly removed when compared to the smooth metal lid. This shows that the suppression of cavity modes is not related to dielectric losses but to the zigzag wire itself. The effect of the losses can be noticed in the level of the $\left|S_{21}\right|$ parameter, but it is very small. Before the starting of the stop band the zigzag lid has worse effect than the metal lid because it allows the excitation of more of the undesired modes, which is a common fact in all artificial stop band surfaces.

We have also measured the relative radiated power of the double-bent microstrip line i) without any lid (open case), ii) packaged with smooth metal lid, and iii) packaged with the two zigzag lids, and these are shown in Fig. 4. The measurements were performed in a reverberation chamber [13] for all the circuits between $4.5 \mathrm{GHz}$ and $6 \mathrm{GHz}$. The radiation 


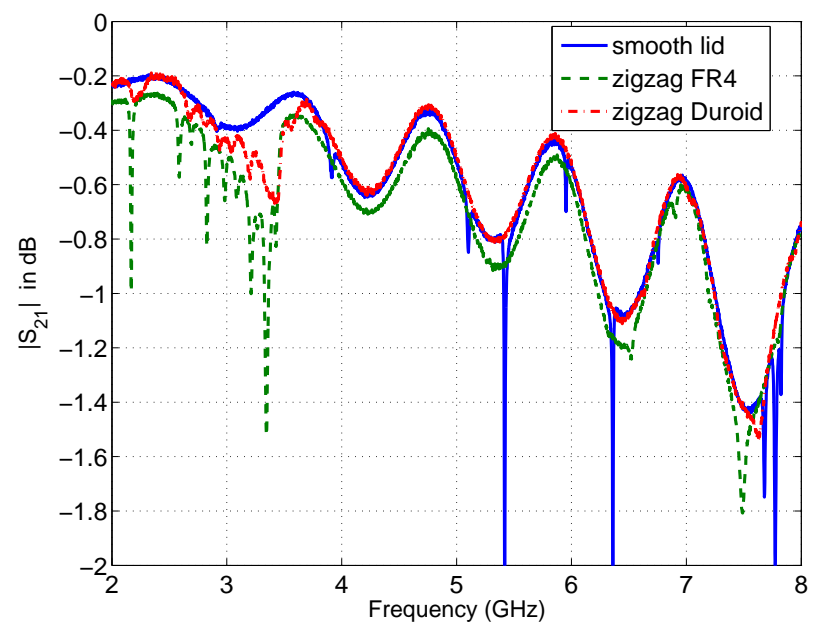

(a)

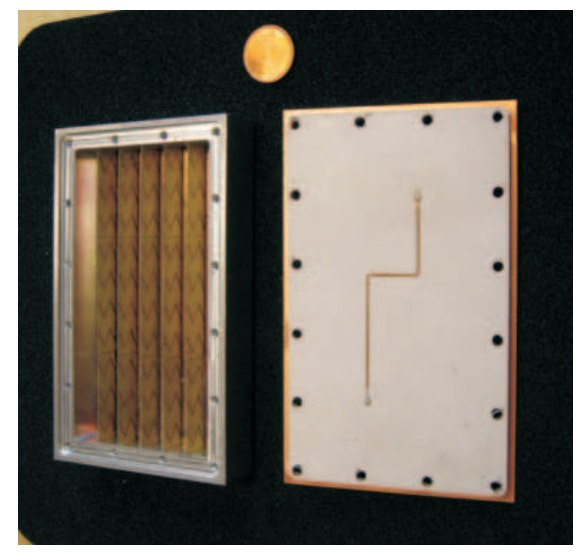

(b)

Fig. 3. Measured S-parameters of microstrip test circuit with different lids (a). Photograph shows test circuit and zigzag lid (b).

loss is clearly very low for the two zigzag cases, compared to the open and smooth lid cases. The lids were held in position during measurements by using just four screws (one per edge).

\section{CONCLUSION}

A new type of periodic surface was proposed to be used for packaging of microstrip circuits below $10 \mathrm{GHz}$. The structure removed cavity modes and radiation as supported by the results. The stopband condition removed also radiation leakage from the small gap between the metal lid and the walls of the box. As a consequence, there is no need for many and tight screws around the rim of lid of the box that package the circuit board.

\section{REFERENCES}

[1] P.-S. Kildal, E. Alfonso, A. Valero-Nogueira, and E. Rajo-Iglesias, "Local metamaterial-based waveguides in gaps between parallel metal plates," IEEE Antennas and Wireless Propagation Letters, vol. 8, pp. 84-87, 2009.

[2] M. Silveirinha, C. Fernandes, and J. Costa, "Electromagnetic characterization of textured surfaces formed by metallic pins," IEEE Transactions on Antennas and Propagation, vol. 56, no. 2, pp. 405-415, Feb. 2008.

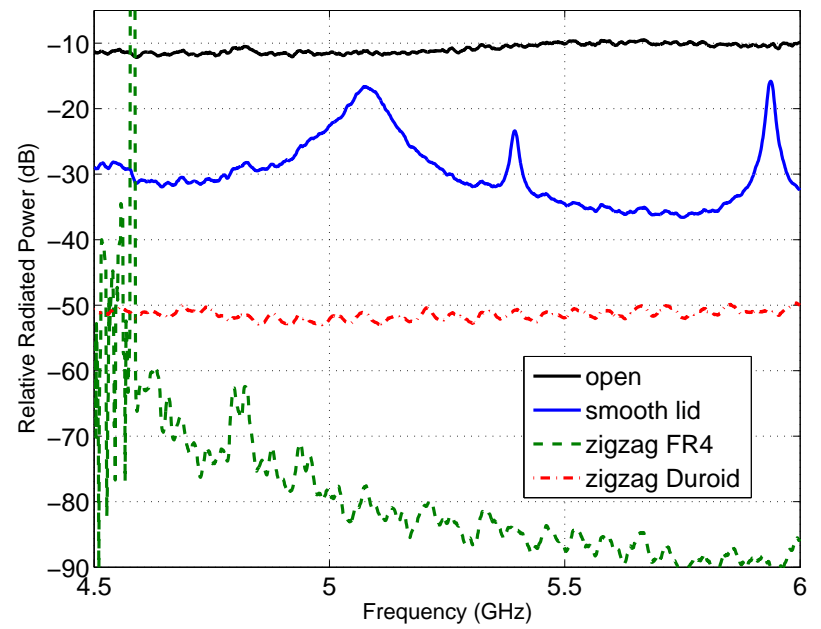

Fig. 4. Relative radiated power measured in reverberation chamber for the microstrip test circuit open, packaged with smooth lid and with the FR4 and Duroid zigzags.

[3] E. Rajo-Iglesias, A. Zaman, and P.-S. Kildal, "Parallel plate cavity mode suppression in microstrip circuit packages using a lid of nails," IEEE Microwave and Wireless Components Letters, vol. 20, no. 1, pp. 31-33, Jan. 2010

[4] E. Rajo-Iglesias and P.-S. Kildal, "Numerical studies of bandwidth of parallel-plate cut-off realised by a bed of nails, corrugations and mushroom-type electromagnetic bandgap for use in gap waveguides," IET Microwaves, Antennas Propagation, vol. 5, no. 3, pp. 282-289, Feb. 2011.

[5] E. Pucci, E. Rajo-Iglesias, and P.-S. Kildal, "New microstrip gap waveguide on mushroom-type EBG for packaging of microwave components," IEEE Microwave and Wireless Components Letters, vol. 22, no. 3, pp. 129-131, March 2012.

[6] M. G. Silveirinha, C. A. Fernandes, J. R. Costa, and C. R. Medeiros, "Experimental demonstration of a structured material with extreme effective parameters at microwaves," Applied Physics Letters, vol. 93, no. 17, pp. $174103-174103-3$, oct 2008.

[7] E. Rajo-Iglesias, P.-S. Kildal, A. Zaman, and A. Kishk, "Bed of springs for packaging of microstrip circuits in the microwave frequency range," IEEE Transactions on Components, Packaging and Manufacturing Technology, vol. 2, no. 10, pp. 1623 -1628, Oct. 2012.

[8] R. Abhari and G. Eleftheriades, "Metallo-dielectric electromagnetic bandgap structures for suppression and isolation of the parallel-plate noise in high-speed circuits," IEEE Transactions on Microwave Theory and Techniques, vol. 51, no. 6, pp. 1629-1639, June 2003.

[9] A. D. Sabata and L. Matekovits, "Electromagnetic band-gap solution for mitigation of parallel-plate noise in power distribution networks," Microwave and Optical Technology Letters, vol. 54, no. 7, pp. 16891692, July 2012.

[10] D. Dawn, Y. Ohashi, and T. Shimura, "A novel electromagnetic bandgap metal plate for parallel plate mode suppression in shielded structures," IEEE Microwave and Wireless Components Letters, vol. 12, no. 5, pp. 166-168, 2002.

[11] Y. Zhang, J. von Hagen, M. Younis, C. Fischer, and W. Wiesbeck, "Planar artificial magnetic conductors and patch antennas," IEEE Transactions on Antennas and Propagation, vol. 51, no. 10, pp. 2704-2712, 2003.

[12] A. Kishk, A. U. Zaman, and P.-S. Kildal, "Numerical prepackaging with PMC lid - efficient and simple design procedure for microstrip circuits including the packaging," ACES Journal, vol. 27, no. 5, pp. 389-398, May 2012.

[13] P. Kildal and K. Rosengren, "Correlation and capacity of MIMO systems and mutual coupling, radiation efficiency, and diversity gain of their antennas: simulations and measurements in a reverberation chamber,' IEEE Communications Magazine, vol. 42, no. 2, pp. 104-112, Feb. 2004. 